Differences of Fetal Heart Rate Patterns between Cephalic and Breech Presentation in Induced Labor

M. Nishijima, H. Tatsumi, K. Amano, S. Ozaki, N. Shimada, M. Arai, K. Osanai

More obstetricians are reluctant to induce labors of breech presentation. We have been managing both cephalic and breech labors by elective induction. In this study evaluated were fetal heart rate patterns in the first stage of breech labor in comparison with those of cephalic labor.

Subject and Method: Randomly selected patients were monitored electronically. Fourty-eight monitor records (28 primiparas and 20 multiparas) in breech group and twenty-six records (10 primiparas and 16 multiparas) in cephalic group were used for comparison. Labors were either induced or augmented in all cases by continuous intravenous infusion of oxytocics with or without artificial rupture of the membranes. Analgesia and anesthesia were given to all patients in the form of balanced analgesia and anesthesia, modified neuroleptanalgesia (diazepam and pentazocine), or epidural anesthesia. FHR was monitored by Corometrics FMS 111, always by direct technic after membrane rupture and by indirect technic before it. Among FHR patterns evaluated were baseline FHR level, baseline long term variability, acceleration, early, variable and late decelerations at the cervical dilatation of less than $5 \mathrm{~cm}, 5$ to $7 \mathrm{~cm}$, and more than $8 \mathrm{~cm}$, respectively. Those three phases of labor were further devided into 20-minute segments for the evaluation of findings. When positive findings of FHR patterns were noted in more than $50 \%$ of the 20-minute segment, the segment was judged positive. The positive segments were expressed in percentage in each phase.

Result: Among variables of the two groups of patients, cevical dilatation and effacement at the time of membrane rupture were significantly more advanced in multiparous breech than in multiparous cephalic group. Otherwise these and station of the presenting part were not statistically different between groups. Significant changes were noted in primiparous breeches with progress of labor in terms of long term variability, early and variable decelerations. Multiparous breeches showed significant changes with labor progression in terms of long term variability, acceleration, and variable deceleration. Among primiparous breeches, significantly lower incidence was noted than among cephalics in terms of long term variability at the cervical dilatation of more than $5 \mathrm{~cm}$, acceleration at less than $5 \mathrm{~cm}$, and early deceleration at 5 to $7 \mathrm{~cm}$. Compared with multiparous cephalics, multiparous breeches showed significantly higher incidence of tachycardia before cervical dilatation of $5 \mathrm{~cm}$, and of long term variability at 5 to $7 \mathrm{~cm}$, while significantly lower incidence of acceleration before $5 \mathrm{~cm}$, and early deceleration after $5 \mathrm{~cm}$.

Comment: Such ominous FHR patterns as variable and late decele-

0300-5577/81/0091-0036\$2.00 Copyright by Walter de Gruyter \& Co. 
rations were not seen more often in breech group than in cephalic group during the first stage of labor. As has been expected, incidence of early deceleration was higher in cephalic than breech group after the cervical dilatation of $5 \mathrm{~cm}$. Lower incidence of long term variability immediately before the 2nd stage in primiparous breech group may indicate that we should be cautious to manage the group in the first stage. However, variability is considered to be influenced by such factors as analgesia and anesthesia. This point remains to be clarified. In the management of breech labor during the first stage, induction or augmentation does not seem to give clinically significant deleterious effect to fetus in terms of FHR patterns.

TACHYCARDIA

Primiparous

Primiparous

Multiparous

Multiparous

Breech

Cephalic

Breech

Cephalic

VARIABILITY

Primiparous

Multiparous

Multiparous

Breech

Cephalic

Breech

Cephalic

ACCELERATION

Primiparous Breech

Primiparous Cephalic

Multiparous Breech

Multiparous Cephalic

EARLY DECELERATION

Primiparous Cephalic

Multiparous Breech

Multiparous Cephalic

VARIABLE DECELERATION

Primiparous Breech

Primiparous Cephalic

Multiparous Breech

Multiparous Cephalic

LATE DECELERATION

Primiparous Breech

Primiparous Cephalic

Multiparous Breech

Multiparous Cephalic
Primiparous Breech

$0.6 \%$
$3.2 \%$
$11.2 \%$
$0 \%$

BEFORE $5 \mathrm{~cm}$

$42.0 \%$

$35.5 \%$

$49.5 \%$

$49.6 \%$

$32.7 \%$

48.4 응

$40.7 \%$

$74.5 \%$

$0 \%$

$4.8 \%$

$0 \%$

$2.0 \%$

$0.6 \%$
$1.6 \%$
$0 \%$
$0 \%$
$1.2 \%$
$0 \%$
$5.0 \%$
$0 \%$

\section{CERVICAL DILATATION}

5 to $7 \mathrm{~cm}$ After $8 \mathrm{~cm}$

$\begin{array}{ll}0 \% & 0 \% \\ 4.8 \% & 14.0 \% \\ 0 \% & 7.7 \% \\ 0 \% & 0 \%\end{array}$

$17.2 \%$

$7.8 \%$

$33.1 \% \quad 20.8 \%$

$47.5 \% \quad 27.4 \%$

$25.8 \% \quad 35.0 \%$

$42.0 \% \quad 35.3 \%$

$51.2 \% \quad 57.1 \%$

$67.3 \% \quad 40.7 \%$

$55.2 \% \quad 38.9 \%$

$2.4 \% \quad 10.0 \%$

$56.0 \% \quad 28.6 \%$

$0 \% \quad 0 \%$

$31.0 \% \quad 22.2 \%$

$0 \% \quad 8.6 \%$

$4.9 \% \quad 14.3 \%$

$4.1 \% \quad 15.4 \%$

$10.3 \% \quad 44.3 \%$

Table 1. The incidence is shown of FHR patterns in primiparous breech and cephalic groups, and those of multiparas at different phases of the first stage of labor.

Nishijima,M., Asst.Prof.M.D.

Kitasato University, Med.School

Dept.Obstet.\& Gynecology

1 Asamizodai, Sagamihara, Kanagawa

Japan 228 
Alterations of the fetal EEG under the influence of labour, hypoxia and analgetics F. KLINK, L. V. KLITZING, R. GROSSPIETZSCH, F. OBERHEUSER The fetal electroencephalogram during labour was performed in order to investigate the effect of labour, hypoxaemia, analgetics and Piracetam on the fetal brain. Figure 1 shows the distribution of the different frequency ranges before (I + II) and after labour activity (III - V).

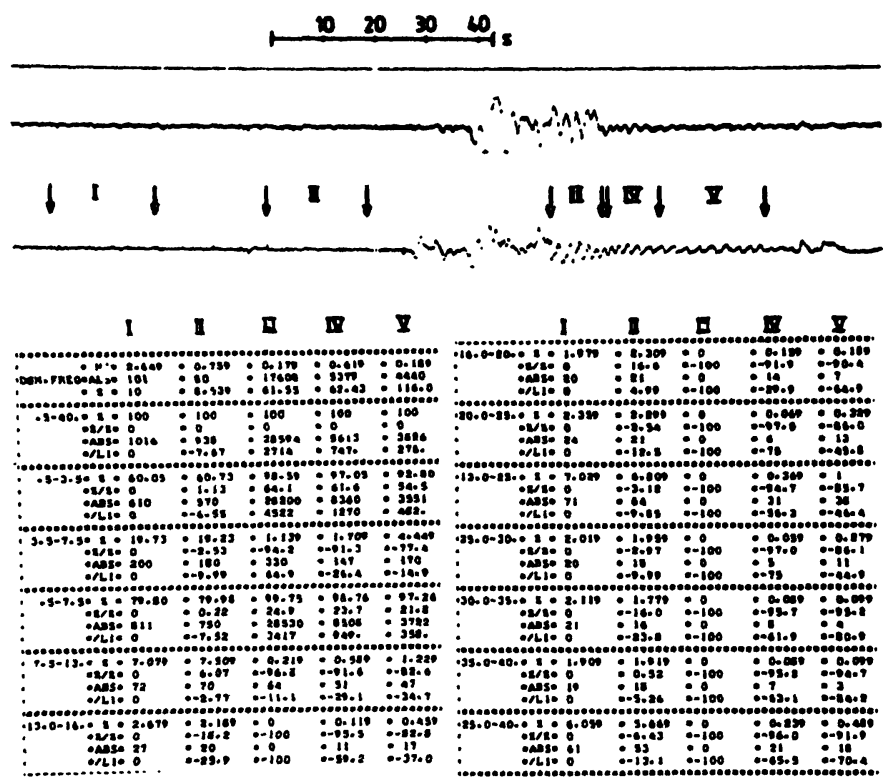

An increase of delta waves can be seen immediately after labour activity and at the same time a decrease of theta waves. Alpha and theta waves change in a similar way. An activation of alpha and beta waves can be observed during labour and low tcpO $\mathrm{CO}_{2} \mathrm{com}-$ bined with late decelerations.

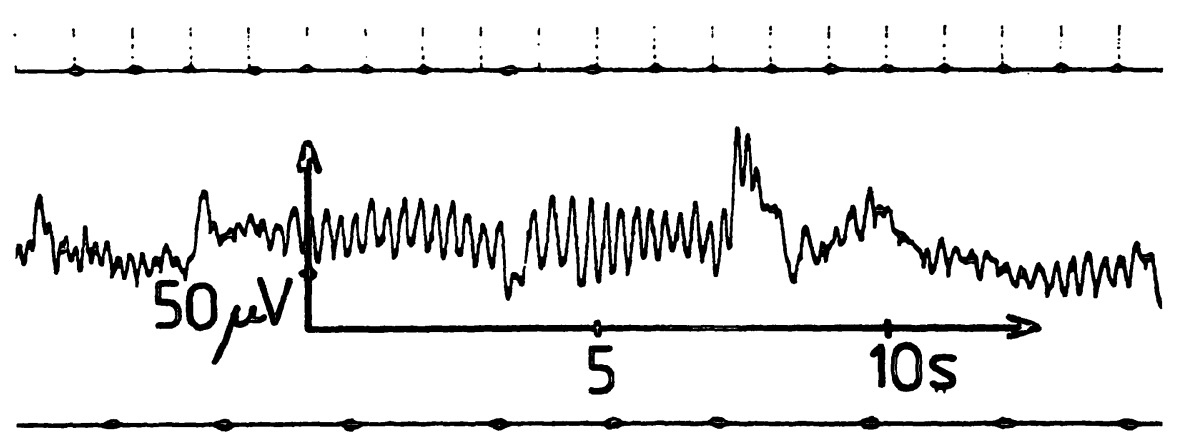

0300-5577/81/0091-0037\$2.00 Copyright by Walter de Gruyter \& Co. 
The application of $75 \mathrm{mg}$ Pethidine to the mother causes $20 \mathrm{~min}$ later a decrease of the amplitude in the fetal EEG of about $50 \%$. The frequency was not significantly changed. Another important question was to examine the influence of $9 \mathrm{~g} / 500 \mathrm{ml} /$ 50 min Piracetam on the fetal EEG. As with respect to the biochemical context of its activity it has been established fo far that:

1. Piracetam increases the ATP/ADP ratio.

2. Piracetam increases the concentration of reduced gluthathione in the brain.

These two processes would be linked with an increased resistance of the brain such as e.g. oxygen deprivation. Our results of long-time EEG evaluations are demonstrated in the following figure representative for this collective.

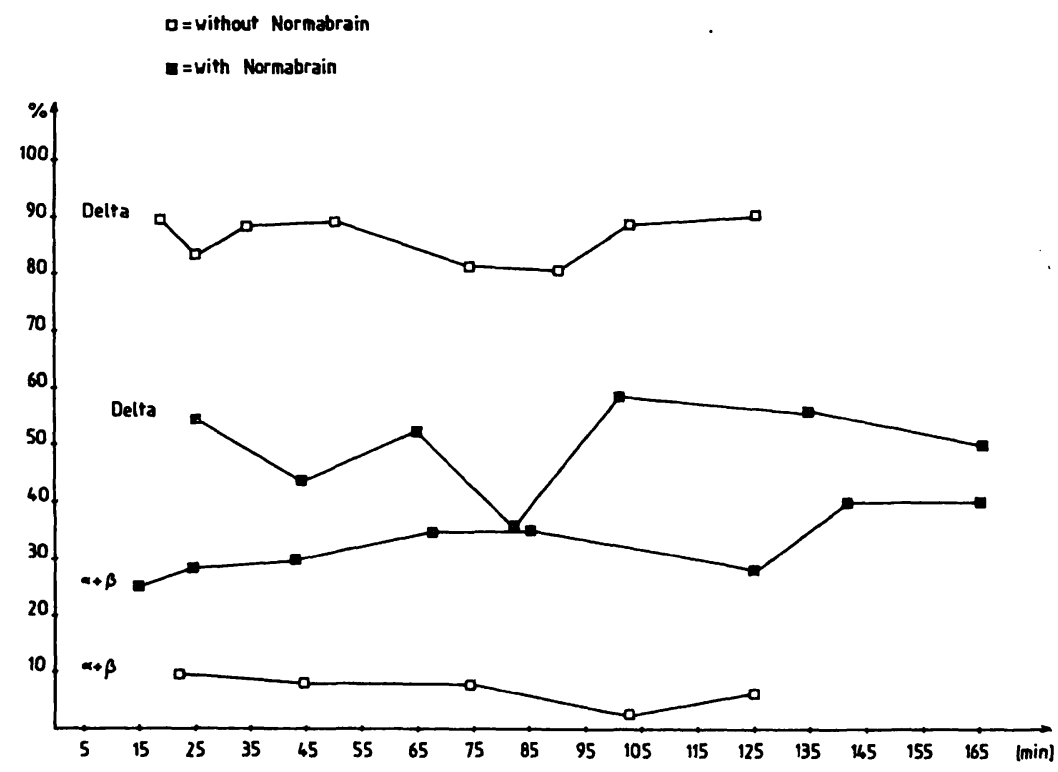

The distribution of frequency reveales an activation of the high frequency EEG signals combined with a decrease of delta waves. These alterations of the EEG are followed by an improvement of the fetal oxygen supply (1).

\section{References}

1. L. V. KLITZING et al.: Computerized analysis of CTG and intrauterine measured $t \mathrm{CpO}_{2}$ of the fetus. (in this journal)

Dr.F.KIink

Abt. Gyn.u.Geburtshile II

der Med.Hochschule

Ratzeburger Allee 160

D-2400 Lïbeck/Germany 